\title{
The Well-Posedness and Stability Analysis of a Computer Series System
}

\author{
Xing Qiao, ${ }^{1}$ Dan Ma, ${ }^{1}$ Fu Zheng, ${ }^{2}$ and Guangtian $\mathrm{Zhu}^{3}$ \\ ${ }^{1}$ School of Mathematical Science, Daqing Normal University, Daqing 163712, China \\ ${ }^{2}$ Department of Mathematics, Bohai University, Jinzhou 121013, China \\ ${ }^{3}$ Academy of Mathematics and System Sciences C.A.S., Beijing 100080, China
}

Correspondence should be addressed to Xing Qiao; xiaoqiao1502@163.com

Received 13 August 2012; Accepted 2 April 2013

Academic Editor: Vu Phat

Copyright (C) 2013 Xing Qiao et al. This is an open access article distributed under the Creative Commons Attribution License, which permits unrestricted use, distribution, and reproduction in any medium, provided the original work is properly cited.

\begin{abstract}
A repairable computer system model which consists of hardware and software in series is established in this paper. This study is devoted to discussing the unique existence of the solution and the stability of the studied system. In view of $c_{0}$ semigroup theory, we prove the existence of a unique nonnegative solution of the system. Then by analyzing the spectra distribution of the system operator, we deduce that the transient solution of the system strongly converges to the nonnegative steady-state solution which is the eigenvector corresponding to eigenvalue 0 of the system operator. Finally, some reliability indices of the system are provided at the end of the paper with a new method.
\end{abstract}

\section{Introduction}

With the development of the modern technology and the extensive use of the electronic products, the reliability problem of the repairable systems has become a hot topic. It is well known that the reliability of a system is an important concept in engineering. The high degree of reliability is usually achieved by introducing redundancy or repairman (e.g., [1$4])$ or applying preventive maintenance (e.g., $[5,6])$, optimal inspection plans (e.g., [7-9]), or optimal replacement policy (e.g., [10]). The aim is to increase the performance of the system by reducing the downtime or the maintenance and inspection cost of the system.

In the general reliability analysis of the computer system, however, because of different characteristics of the hardware and software, we cannot simply take the hardware and software as a unit or two different types of units [9]. Then, it is rare to analyze synthetically [11]. With the passage of the using time and the number of failures increasing, the reliability of the hardware would descend, and the repair time would be longer [12]. During the software debugging and testing stages, as the failures occur, potential software error is discovered and corrected constantly which make the software reliability grow [13]. Since the hardware failure or software failure leads to the whole computer system failure, the computer system can be formulated as a series system with hardware and software (namely, hardware and software in series). There are some obstacles to overcome to obtain the main result since our model is more complicated than that of [11-13].

In this paper, we study a repairable computer system which is composed of hardware and software in series. The unique existence of the system solution is obtained by using $c_{0}$ semigroup theory. The exponential stability of the system is further achieved by analyzing the spectrum distribution of the system operator given by (2)-(4), which shows that the solution to the system (2)-(4) is exponentially stable. Thus we not only provide strict theoretical foundation for reliability study but also make it more valuable in practice.

The remainder of the paper is organized as follows. In Section 2 we formulate the mathematical model of the system with concerned notations; in Section 3.1 we show the unique existence of the dynamic solution of the system. In Section 3.2 we study the unique existence of the solution of the abstract Cauchy problem corresponding to the system and present a detailed spectral analysis of the system operator; some steady-state reliability indices of the system are presented in Section 4 , and Section 5 concludes the paper. 


\section{Mathematical Model Formulation}

In the reliability analysis of repair system, it is usually assumed that the repaired units which compose system are as good as new, and the failed units are repaired immediately. However, in reality it is usually not the case. In real life, it is possible that the reliability reduces after the software failure each time. That is, the condition $F_{S}^{(n)}(t)=F_{S}\left(a^{n-1} t\right)=1-$ $e^{-a^{n-1} \lambda_{s} t}, t \geq 0, \lambda_{s}>0$, and the coefficient $a>1$. With the number of repair times increasing, the failure rate is increasing gradually. In view of the aging and accumulative wear, the repair time will become longer and longer and tend towards infinity; that is, the system is nonrepairable. Therefore, we first suppose that the software is overhauled (replaced) to be as good as new after the $(N-1)$ th minimal repair, and studying the number of minimal repair before overhaul repair is more appropriate. And we will also discuss how its reliability will be affected by the number of minimal repair and overhaul. In [14], the author supposed that the software cannot be repaired as good as new and utilized the geometric process and supplementary variable technique to analyze the system reliability. However, the life and repair times of the hardware and software are supposed to follow exponential distribution. In this paper, under assumption that the life time of the hardware and software follows exponential distribution and repair time is subject to the general distribution, we set up a mathematical model of the repairable computer system by the supplementary variable method, which is composed of the hardware and software in series. The hardware is repaired to be as good as new, the software is repaired periodically, and restored software life decreases. After a period of time, an overhaul makes it as a new one.

The system model is formulated specifically as follows.

(i) The computer system is composed of hardware $H$ and software $S$ in series.

(ii) The distribution function of the life time $X_{H}$ of hardware $H$ is $F_{H}(t)=1-e^{-\lambda_{h} t}, t \geq 0, \lambda_{h}>0$.

(iii) The distribution function of the life time $X_{S}^{(n)}$ of software $S$ during its $n$th period (e.g., the time between the completion of its $(n-1)$ th repair and that of the $n$th repair) is $F_{S}^{n}(t)=F_{S}\left(a^{n-1} t\right)=1-e^{-a^{n-1} \lambda_{s} t}, t \geq 0$, $\lambda_{s}>0, a>1, n=1,2, \ldots, N$.

(iv) Let $Y_{1}$ be the repair time of hardware $H, Y_{2}$ the repair time of software $S$ after its $n$th failure $(n=1,2, \ldots, N-$ 1 ), and $Y_{3}$ the repair time of software $S$ after its $n$th failure, respectively. Their distribution functions are $G_{j}(t)=\int_{0}^{t} g_{j}(x) d x=1-e^{-\int_{0}^{t} \mu_{j}(x) d x}$ and $E\left[Y_{j}\right]=1 / \mu_{j}$, $j=1,2,3$, where $\mu_{3}(x)>\mu_{2}(x)$, for all $x \geq 0$.

(v) The hardware $H$ is repaired as good as new. The software $S$ is performed by a minimal repair (e.g., a maintenance action performed on a failed system by which its survival time is decreasing) during its $n$th $(n=1,2, \ldots, N-1)$ period and an overhaul repair (e.g., a maintenance action performed on a failed system by which it is repaired as good as new) during its $N$ th period. The above stochastic variables are independent of each other.

Let $N(t)$ be the system state at time $t$, and assume all the possible states as below.

$0 i(i=1,2, \ldots, N)$ the system is working.

$1 i(i=1,2, \ldots, N)$ the system is failed because the failure hardware $H$ is being repaired in the $i$ th time.

$2 i(i=1,2, \ldots, N)$ the system has in failure state because the failure software $S$ is being repaired minimally in the $i$ th time $(i=1,2, \ldots, N-1)$ and the software $S$ is being overhauled in the $N$ th time.

Then the system state space is $E=\{0 i, 1 i, 2 i\} \quad(i=$ $1,2, \ldots, N)$, in which the working state space is $W=\{0 i\}$ $(i=1,2, \ldots, N)$ and the failure state space is $F=\{1 i, 2 i\}$ $(i=1,2, \ldots, N)$.

When $N(t)=j i(j=0,1,2 ; i=1,2, \ldots, N)$ supplement variable $Y_{j}(t)(j=1,2,3)$ which denotes the elapsed repair time of hardware $H$, the elapsed minimal repair time of software $S$, and its elapsed overhaul time at time $t$, respectively, then $\left\{N(t), Y_{j}(t)\right\}$ constitutes a matrix Markov process whose state probabilities are defined as follows:

$$
\begin{array}{r}
P_{0 i}(t)=P\{N(t)=0 i\}, \quad i=1,2, \ldots, N, \\
P_{1 i}(x, t)=P\left\{x<Y_{1}(t) \leq x+d x, N(t)=1 i\right\}, \\
i=1,2, \ldots, N, \\
P_{2 i}(x, t)=P\left\{x<Y_{2}(t) \leq x+d x, N(t)=2 i\right\}, \\
i=1,2, \ldots, N-1, \\
P_{2 N}(x, t)=P\left\{x<Y_{3}(t) \leq x+d x, N(t)=2 N\right\} .
\end{array}
$$

Then by using the method of probability analysis, the system under consideration can be formulated as the following equations:

$$
\begin{gathered}
\frac{d P_{01}(t)}{d t}=-\left(\lambda_{h}+\lambda_{s}\right) P_{01}(t)+\int_{0}^{\infty} P_{11}(x, t) \mu_{1}(x) d x \\
\quad+\int_{0}^{\infty} P_{2 N}(x, t) \mu_{3}(x) d x \\
\frac{d P_{0 i}(t)}{d t}=-\left(\lambda_{h}+a^{i-1} \lambda_{s}\right) P_{0 i}(t)+\int_{0}^{\infty} P_{1 i}(x, t) \mu_{1}(x) d x \\
+\int_{0}^{\infty} P_{2(i-1)}(x, t) \mu_{2}(x) d x, \\
i=2,3, \ldots, N, \\
\frac{\partial P_{1 i}(x, t)}{\partial x}+\frac{\partial P_{1 i}(x, t)}{\partial t}=-\mu_{1}(x) P_{1 i}(x, t), \\
\frac{\partial P_{2 i}(x, t)}{\partial x}+\frac{\partial P_{2 i}(x, t)}{\partial t}=-\mu_{2}(x) P_{2 i}(x, t), \\
\quad i=1,2, \ldots, N-1, N, \\
\frac{\partial P_{2 N}(x, t)}{\partial x}+\frac{\partial P_{2 N}(x, t)}{\partial t}=-\mu_{3}(x) P_{2 N}(x, t) .
\end{gathered}
$$


The boundary conditions are

$$
\begin{gathered}
P_{1 i}(0, t)=\lambda_{h} P_{0 i}(t), \quad i=1,2, \ldots, N, \\
P_{2 i}(0, t)=a^{i-1} \lambda_{s} P_{0 i}(t), \quad i=1,2, \ldots, N .
\end{gathered}
$$

The initial conditions are

$$
P_{01}(0)=1 \text {, and the others are equal to } 0 .
$$

Taking into account the practical background, we assume that

$$
\begin{gathered}
0<K=\sup _{x \in[0, \infty)} \mu_{j}(x)<\infty, \\
\int_{0}^{T} \mu_{j}(x) d x<\infty, \quad 0<T<\infty, \\
\int_{0}^{\infty} \mu_{j}(x) d x=\infty, \quad j=1,2,3 .
\end{gathered}
$$

And then, we may know that many repairs/services are done periodically in practice. So we can suppose that the mean of repair/service rate exists and does not equal to 0 $([15,16])$ :

$$
0<\mu_{j}=\lim _{x \rightarrow \infty} \frac{1}{x} \int_{0}^{x} \mu_{j}(\tau) d \tau<\infty, \quad j=1,2,3 .
$$

\section{Stability Analysis}

In this section, we firstly study the unique existence of the classical solution of the system by pure analysis method in Section 3.1. In Section 3.2, we will formulate the problem into a suitable Banach space. Then we explain that the system has a unique generalized solution, and it is just the classical one when $t>0$. We also carry out a detailed spectral analysis of the system operator $A+E$. Finally, the exponential stability of the system can be readily achieved.

\subsection{Unique Existence of the Classical Solution}

Theorem 1. The system (2)-(4) has a unique nonnegative solution in $C[0, T]$, for any $T>0$.

Proof. Solving (2)-(4) with the method in [17] one gets

$$
\begin{aligned}
P_{01}(t)= & e^{-\pi_{1} t}+\int_{0}^{\infty} k_{11}(t-\eta) P_{11}(0, \eta) d \eta \\
& +\int_{0}^{\infty} k_{2 N}(t-\eta) P_{2 N}(0, \eta) d \eta, \\
P_{0 i}(t)= & e^{-\pi_{i} t}+\int_{0}^{\infty} k_{1 i}(t-\eta) P_{1 i}(0, \eta) d \eta \\
& +\int_{0}^{\infty} k_{2(i-1)}(t-\eta) P_{2(i-1)}(0, \eta) d \eta, \\
P_{11}(0, t)= & \lambda_{h}\left(e^{-\pi_{1} t}+\int_{0}^{\infty} k_{11}(t-\eta) P_{11}(0, \eta) d \eta\right. \\
& \left.\quad+\int_{0}^{\infty} k_{2 N}(t-\eta) P_{2 N}(0, \eta) d \eta\right),
\end{aligned}
$$

$$
\begin{aligned}
& P_{1 i}(0, t)=\lambda_{h}\left(e^{-\pi_{i} t}+\int_{0}^{\infty} k_{1 i}(t-\eta) P_{1 i}(0, \eta) d \eta\right. \\
& \left.+\int_{0}^{\infty} k_{2(i-1)}(t-\eta) P_{2(i-1)}(0, \eta) d \eta\right) \\
& i=2,3, \ldots, N \text {, } \\
& P_{21}(0, t)=\lambda_{s}\left(e^{-\pi_{1} t}+\int_{0}^{\infty} k_{11}(t-\eta) P_{11}(0, \eta) d \eta\right. \\
& \left.+\int_{0}^{\infty} k_{2 N}(t-\eta) P_{2 N}(0, \eta) d \eta\right) \\
& P_{2 i}(0, t)=a^{i-1} \lambda_{s}\left(e^{-\pi_{i} t}+\int_{0}^{\infty} k_{1 i}(t-\eta) P_{1 i}(0, \eta) d \eta\right. \\
& \left.+\int_{0}^{\infty} k_{2(i-1)}(t-\eta) P_{2(i-1)}(0, \eta) d \eta\right) \\
& i=2,3, \ldots, N \text {, }
\end{aligned}
$$

where

$$
\begin{gathered}
\pi_{i}=\lambda_{h}+a^{i-1} \lambda_{s}, \\
k_{1 i}(t-\eta)=\int_{0}^{t-\eta} e^{-\pi_{i}(t-\eta) \pi_{i} v-\int_{0}^{v} \mu_{1}(\tau) d \tau} \mu_{1}(v) d v, \\
i=1,2, \ldots, N, \\
k_{2 i}(t-\eta)=\int_{0}^{t-\eta} e^{-\pi_{i+1}(t-\eta) \pi_{i+1} v-\int_{0}^{v} \mu_{2}(\tau) d \tau} \mu_{2}(v) d v, \\
i=1,2, \ldots, N-1, \\
k_{2 N}(t-\eta)=\int_{0}^{t-\eta} e^{-\pi_{1}(t-\eta) \pi_{1} v-\int_{0}^{v} \mu_{3}(\tau) d \tau} \mu_{3}(v) d v .
\end{gathered}
$$

With the help of (7), we can get the following convolution-type integral equation

$$
P(t)=F(t)+\int_{0}^{t} K(t-\eta) P(\eta) d \eta,
$$

where

$$
\begin{aligned}
& P(t)=\left(P_{01}(t), \ldots, P_{0 N}(t), P_{11}(0, t), \ldots, P_{1 N}(0, t),\right. \\
& \left.P_{21}(0, t), \ldots, P_{2 N}(0, t)\right)^{T} \text {, } \\
& F(t)=\left(f(t), \lambda_{h} f(t), \lambda_{s}\left(e^{-\pi_{1} t}, a e^{-\pi_{2} t}, \ldots, a^{N-1} e^{-\pi_{N} t}\right)^{T},\right. \\
& f(t)=\left(e^{-\pi_{1} t}, e^{-\pi_{2} t}, \ldots, e^{-\pi_{N} t}\right), \\
& K(t-\eta)=\left(\begin{array}{lll}
O_{n \times n} & K^{11}(t-\eta) & K^{12}(t-\eta) \\
O_{n \times n} & K^{21}(t-\eta) & K^{22}(t-\eta) \\
O_{n \times n} & K^{31}(t-\eta) & K^{32}(t-\eta)
\end{array}\right) \\
& K^{12}(t-\eta) \\
& =\left(\begin{array}{cccc}
0 & \cdots & 0 & k_{2 N}(t-\eta) \\
k_{21}(t-\eta) & \cdots & 0 & 0 \\
\vdots & \ddots & \vdots & \vdots \\
0 & \cdots & k_{2(N-1)}(t-\eta) & 0
\end{array}\right)_{n \times n},
\end{aligned}
$$




$$
\begin{aligned}
& K^{22}(t-\eta)=\lambda_{h} K^{12}(t-\eta) \\
& K^{32}(t-\eta) \\
& =\left(\begin{array}{cccc}
0 & \cdots & 0 & \lambda_{s} k_{2 N}(t-\eta) \\
a \lambda_{s} k_{21}(t-\eta) & \cdots & 0 & 0 \\
\vdots & \ddots & \vdots & \vdots \\
0 & \cdots & a^{N-1} \lambda_{s} k_{2(N-1)}(t-\eta) & 0
\end{array}\right)_{n \times n}, \\
& K^{11}(t-\eta)=\operatorname{diag}\left(k_{11}(t-\eta), k_{11}(t-\eta), \ldots,\right. \\
& \left.k_{11}(t-\eta)\right)_{n \times n} \\
& K^{21}(t-\eta)=\operatorname{diag}\left(\lambda_{h} k_{11}(t-\eta), \lambda_{h} k_{11}(t-\eta), \ldots,\right. \\
& \left.\lambda_{h} k_{11}(t-\eta)\right)_{n \times n}, \\
& K^{31}(t-\eta)=\operatorname{diag}\left(\lambda_{s} k_{11}(t-\eta), a \lambda_{s} k_{11}(t-\eta), \ldots,\right. \\
& \left.a^{N-1} \lambda_{s} k_{11}(t-\eta)\right)_{n \times n} \text {. }
\end{aligned}
$$

It is clear that the unique existence of the nonnegative solution of the system (2)-(4) is equal to that of the convolution-type integral equation (9). Since, for any $T>0$, each coordinate of $F(t)$ and $K(t-\eta)$ is nonnegatively bounded function and is absolutely integrable in $C[0, T]$, we can obtain that the convolution-type integral equation (9) has a unique nonnegative solution in $C[0, T]$ by using the related theory in integral equation. For this reason, the system (2)-(4) has a unique nonnegative solution in $C[0, T]$, for any $T>0$. The proof is complete.

3.2. Exponential Stability. In this subsection, in order to further study the properties of the studied system, we will formulate the problem into a suitable Banach space. Then we study the unique existence of its solution and explain the exponential stability of the system by analyzing the spectrum distribution of the system operator in detail.

Firstly, let the state space $X$ be

$$
\begin{aligned}
X=\{P & \in R^{n} \times\left(L^{1}\left(R^{+}\right) \times L^{1}\left(R^{+}\right)\right)^{n} \mid\|P\| \\
& \left.=\left|P^{0}\right|+\sum_{i=1}^{2}\left\|P^{i}\right\|<\infty\right\},
\end{aligned}
$$

where

$$
\begin{gathered}
P=\left(P^{0}, P^{1}(x), P^{2}(x)\right), \\
P^{0}=\left(P_{01}, P_{02}, \ldots, P_{0 N}\right)^{T}, \\
P^{1}(x)=\left(P_{11}(x), P_{12}(x), \ldots, P_{1 N}(x)\right)^{T}, \\
P^{2}(x)=\left(P_{21}(x), P_{22}(x), \ldots, P_{2 N}(x)\right)^{T}, \\
\left|P^{0}\right|=\sum_{i=1}^{N}\left|P_{0 i}\right|, \quad\left|P^{j}\right|=\sum_{i=1}^{N}\left\|P_{j i}\right\|_{L^{1}\left(R^{+}\right)}, \quad j=1,2 .
\end{gathered}
$$

It is obvious that $X$ is a Banach space.

Secondly, we will introduce some operators in $X$.
Consider $A P=\left(\operatorname{diag}\left(-\left(\lambda_{h}+\lambda_{s}\right),-\left(\lambda_{h}+a \lambda_{s}\right), \ldots,-\left(\lambda_{h}+\right.\right.\right.$ $\left.\left.a^{N-1} \lambda_{s}\right)\right) P^{0}, \operatorname{diag}\left(-(d / d x)-\mu_{1}(x), \ldots,-(d / d x)-\mu_{1}(x)\right) P^{1}(x)$, $\operatorname{diag}\left(-(d / d x)-\mu_{2}(x), \ldots,-(d / d x)-\mu_{2}(x),-(d / d x)-\right.$ $\left.\left.\mu_{3}(x)\right) P^{2}(x)\right)$.

Taking $D(A)=\left\{P=\left(P^{0}, P^{1}(x), P^{2}(x)\right) \in X \mid\left(d P_{j i}(x) /\right.\right.$ $d x) \in L^{1}\left(R^{+}\right), j=1,2, i=1,2, \ldots, N, P_{j i}(x)(j=1,2, i=1,2$, $\ldots, N)$ are absolutely continuous functions satisfying $P(0)=$ $\left.\left(P^{0}, P^{1}(0), P^{2}(0)\right)=\left(P^{0}, \lambda_{h} P^{0}, \lambda_{s}\left(P_{01}, a P_{02}, \ldots, a^{N-1} P_{0 N}\right)^{T}\right)\right\}$,

$$
E P=\left(\begin{array}{ccc}
O_{n \times n} & E_{1} & E_{2} \\
O_{2 n \times n} & O_{2 n \times n} & O_{2 n \times n}
\end{array}\right)\left(\begin{array}{c}
P^{0} \\
P^{1}(x) \\
P^{2}(x)
\end{array}\right), \quad D(E)=X,
$$

where $O_{n \times n}, O_{2 n \times n}$ are zero vectors and $E_{1}=\operatorname{diag}\left(\mu_{1}(x)\right.$, $\left.\mu_{1}(x), \ldots, \mu_{1}(x)\right)_{n \times n}$,

$E_{2}$

$$
=\left(\begin{array}{ccccc}
0 & 0 & \cdots & 0 & \int_{0}^{\infty} \mu_{3}(x) d x \\
0 & \int_{0}^{\infty} \mu_{2}(x) d x & \cdots & 0 & 0 \\
\vdots & \vdots & \ddots & \vdots & \vdots \\
0 & 0 & \cdots & \int_{0}^{\infty} \mu_{2}(x) d x & 0
\end{array}\right)_{n \times n}
$$

Then the former equations (2)-(4) can be formulated as an abstract Cauchy problem into the suitable Banach space $X$, that is,

$$
\begin{gathered}
\frac{d}{d t} P(\cdot, t)=(A+E) P(\cdot, t), \quad t \geq 0, \\
P(\cdot, t)=\left(P^{0}(t), P^{1}(\cdot, t), P^{2}(\cdot, t)\right), \\
P(\cdot, 0)=P_{0}=\left((1,0, \ldots, 0)_{1 \times n}^{T}, O_{n \times 1}, O_{n \times 1}\right) .
\end{gathered}
$$

Since the system (2)-(4) is rewritten as an abstract Cauchy problem, it is necessary to prove the well-posedness of the system (15). Next, we will prove that the system (15) has a unique nonnegative solution by using $c_{0}$ semigroup theory. We present the expression of the dynamic solution of the system equation.

For convenience, we will present four useful lemmas.

Lemma 2. There exists constant $L>0$ such that for any $t>0$ (see [18])

$$
\int_{t}^{\infty} e^{-\int_{t}^{x} \mu_{j}(\tau) d \tau} d x \leq L, \quad j=1,2,3 .
$$

Lemma 3. For any $r \in\{r \in \mathbb{C} \mid \operatorname{Re} r>0$ or $r=i a, a \in$ $R, a \neq 0\}$ (see [18]),

$$
\left|\int_{0}^{\infty} \mu_{j}(x) e^{-\int_{0}^{x}\left(r+\mu_{j}(\tau)\right) d \tau} d x\right|<1, \quad j=1,2,3 .
$$

Lemma 4. The system operator $A+E$ is a dispersive operator (see [19]) with dense domain.

The proof of Lemma 4 can be seen in [20-22]. 
Lemma 5. $\{r \in \mathbb{C} \mid \operatorname{Re} r>0$ or $r=i a, a \in R \backslash\{0\}\} \subseteq \rho(A+E)$.

Proof. Firstly, for any $G=\left(g^{0}, g^{1}(x), g^{2}(x)\right) \in X$, here $g^{j}=$ $\left(g_{j 1}, g_{j 2}, \ldots, g_{j N}\right)^{T}, j=0,1,2$, considering $[r I-(A+E)] P=$ $G$. That is,

$$
\begin{gathered}
\left(r+\lambda_{h}+\lambda_{s}\right) P_{01}-\int_{0}^{\infty} \mu_{1}(x) P_{11}(x) d x \\
-\int_{0}^{\infty} \mu_{3}(x) P_{2 N}(x) d x=g_{01}, \\
\left(r+\lambda_{h}+a^{j-1} \lambda_{s}\right) P_{0 j}-\int_{0}^{\infty} \mu_{1}(x) P_{1 j}(x) d x \\
-\int_{0}^{\infty} \mu_{2}(x) P_{2(j-1)}(x) d x=g_{0 j}, \\
j=2, \ldots, N, \\
\frac{d}{d x} P_{1 j}(x)+\left(r+\mu_{1}(x)\right) P_{1 j}(x)=g_{1 j}(x), 2, \ldots, N, \\
\frac{d}{d x} P_{2 j}(x)+\left(r+\mu_{2}(x)\right) P_{2 j}(x)=g_{2 j}(x), \\
j=2,3, \ldots, N-1, \\
\frac{d}{d x} P_{2 N}(x)+\left(r+\mu_{3}(x)\right) P_{2 N}(x)=g_{2 N}(x) .
\end{gathered}
$$

And we can suppose

$$
\begin{gathered}
P_{1 j}(0)=\lambda_{h} P_{0 j}, \quad j=1,2, \ldots, N, \\
P_{2 j}(0)=a^{j-1} \lambda_{s} P_{0 j}, \quad j=1,2, \ldots, N .
\end{gathered}
$$

Solving (20)-(22) with the help of (23) one gets

$$
\begin{array}{r}
P_{1 j}(x)=P_{1 j}(0) e^{-\int_{0}^{x}\left[r+\mu_{1}(\eta)\right] d \eta}+\int_{0}^{x} e^{-\int_{\tau}^{x}\left[r+\mu_{1}(\eta)\right] d \eta} g_{1 j}(\tau) d \tau, \\
j=1,2, \ldots, N, \\
P_{2 j}(x)=P_{2 j}(0) e^{-\int_{0}^{x}\left[r+\mu_{2}(\eta)\right] d \eta}+\int_{0}^{x} e^{-\int_{\tau}^{x}\left[r+\mu_{2}(\eta)\right] d \eta} g_{2 j}(\tau) d \tau, \\
j=1,2, \ldots, N-1, \\
P_{2 N}(x)=P_{2 N}(0) e^{-\int_{0}^{x}\left[r+\mu_{3}(\eta)\right] d \eta}+\int_{0}^{x} e^{-\int_{\tau}^{x}\left[r+\mu_{3}(\eta)\right] d \eta} g_{2 N}(\tau) d \tau .
\end{array}
$$

Noticing that $g_{j i}(x) \in L^{1}[0, \infty), j=1,2, i=1,2, \ldots, N$, together with Lemma 2 , we know $P_{j i}(x) \in L^{1}[0, \infty), j=1,2$, $i=1,2, \ldots, N$. This implies that $[r I-(A+E)]$ is an onto mapping.

Secondly, we will prove that this operator is also an injective mapping. That is, the operator equation $[r I-(A+$ $E)] P=0$ has a unique solution 0 . Set $G=0$ in the former discussion. Then we can obtain the following matrix equation by combing (18)-(19) with (24):

$$
\begin{gathered}
\left(\begin{array}{ccccc}
r+b_{1} & 0 & \cdots & 0 & -a^{N-1} \lambda_{s} W_{3} \\
-\lambda_{s} W_{2} & r+b_{2} & \cdots & 0 & 0 \\
0 & -a \lambda_{s} W_{2} & \ddots & 0 & 0 \\
\vdots & \vdots & \ddots & \vdots & \vdots \\
0 & 0 & \cdots & r+b_{N-1} & 0 \\
0 & 0 & \cdots & -a^{N-2} \lambda_{s} W_{2} & r+b_{N}
\end{array}\right) \\
\times\left(\begin{array}{c}
P_{01} \\
P_{02} \\
P_{03} \\
\vdots \\
P_{0(N-1)} \\
P_{0 N}
\end{array}\right)=\left(\begin{array}{c}
0 \\
0 \\
0 \\
\vdots \\
0 \\
0
\end{array}\right),
\end{gathered}
$$

where

$$
\begin{aligned}
& b_{j}=\lambda_{h}\left(1-W_{1}\right)+a^{j-1} \lambda_{s} \quad(j=1,2, \ldots, N), \\
& W_{j}=\int_{0}^{\infty} \mu_{j}(x) e^{-\int_{0}^{x}\left[r+\mu_{j}(\tau)\right] d \tau} d x, \quad j=1,2,3 .
\end{aligned}
$$

It is clear that

$$
\begin{aligned}
\left|r+b_{j}\right| & =\left|r+\lambda_{h}\left(1-W_{1}\right)+a^{j-1} \lambda_{s}\right|>\left|r+a^{j-1} \lambda_{s}\right| \\
& >\left|a^{j-1} \lambda_{s}\right|>\left|-a^{j-1} \lambda_{s} W_{2}\right|, \quad j=1,2, \ldots, N-1, \\
\left|r+b_{N}\right| & =\left|r+\lambda_{h}\left(1-W_{1}\right)+a^{N-1} \lambda_{s}\right|>\left|r+a^{N-1} \lambda_{s}\right| \\
& >\left|a^{N-1} \lambda_{s}\right|>\left|-a^{N-1} \lambda_{s} W_{3}\right|>0,
\end{aligned}
$$

for $r>0$ or $r=i a, a \in R \backslash\{0\}$. From Lemma 3, we can obtain $\left|W_{j}\right|<1, j=1,2,3$. Thus the matrix of coefficients of the linear equations (25) is a strictly diagonal-dominant matrix about column. Therefore, this matrix is invertible, which manifests that operator $[r I-(A+E)]$ is a one-to-one mapping.

Because $[r I-(A+E)]$ is densely defined closed in $X$, we can derive that $[r I-(A+E)]^{-1}$ exists and is bounded by recalling inverse operator theorem and closed graph theorem. That is, set $\{r \in \mathbb{C} \mid \operatorname{Re} r>0$ or $r=i a, a \in R \backslash\{0\}\}$ belongs to the resolvent set of the system operator $A+E$. Thus we complete the proof of Lemma 5.

Theorem 6. The simple eigenvalue of system operator $A+E$ is 0 .

Proof. Firstly, we will explain that 0 is the eigenvalue of $A+E$ with positive eigenvector.

Consider $(A+E) P=0$ and assume that $P$ satisfies the boundary conditions (23). Then repeating the proof process 
of the injective mapping in Lemma 5 with $r=0$, we can get the following solutions:

$$
\begin{gathered}
P_{0 j}=a^{N-j} P_{0 N} \quad(j=1,2, \ldots, N), \\
P_{1 j}(x)=a^{N-j} \lambda_{h} P_{0 N} e^{-\int_{0}^{x} \mu_{1}(\eta) d \eta} \quad(j=1,2, \ldots, N), \\
P_{2 j}(x)=a^{N-j} \lambda_{s} P_{0 N} e^{-\int_{0}^{x} \mu_{2}(\eta) d \eta} \quad(j=1,2, \ldots, N-1), \\
P_{2 N}(x)=a^{N-1} \lambda_{s} P_{0 N} e^{-\int_{0}^{x} \mu_{3}(\eta) d \eta},
\end{gathered}
$$

where $P_{0 N}$ is an arbitrary real number. Then it can be derived that $P_{j i}(x), j=1,2, i=1,2, \ldots, N$, for all $x \in[0, \infty)$ by taking $P_{0 N}>0$ without loss of generality. Since the vector

$$
\begin{aligned}
P^{*}= & \left(\left(P_{01}^{*}, \ldots, P_{0 N}^{*}\right)^{T},\left(P_{11}^{*}(x), \ldots, P_{1 N}^{*}(x)\right)^{T},\right. \\
& \left.\left(P_{21}^{*}(x), \ldots, P_{2 N}^{*}(x)\right)^{T}\right)
\end{aligned}
$$

is the positive eigenvector corresponding to eigenvalue 0 of the system operator $A+E$ and it is also the positive steadystate solution of the system, here $P_{0 i}^{*}$ and $P_{j i}^{*}(x)$, respectively, signify $P_{0 i}$ and $P_{j i}(x)$ showed in $(28), j=1,2, i=1,2, \ldots, N$. In addition, it is easy to see that the geometric multiplicity of eigenvalue 0 in $X$ is one.

Secondly, we will explain that the algebraic multiplicity of eigenvalue 0 is one.

Taking vector $Q=\left(I_{n \times 1}^{0}, I_{n \times 1}^{1}, I_{n \times 1}^{2}\right)$, here $I^{j}=(1,1, \ldots$, $1)^{T}, j=0,1,2$. Then $Q \in X^{*}$. For any $P \in D(A+E)$, it is not difficult to show that $\langle(A+E) P, Q\rangle=0$ by noticing the boundary conditions. Therefore, we can deduce that $\langle P,(A+$ $\left.E)^{*} Q\right\rangle=0$, for all $P \in X$, for $D(A)$ is dense in $X$. This manifests that $Q$ is the eigenvector of $(A+E)^{*}$, the adjoint operator of $A+E$, corresponding to eigenvalue 0 .

In the light of $[23,24]$, we only need to explain that the algebraic index of 0 in $X$ is one. We use the reduction to absurdity. Assume that the algebraic index of 0 is 2 without loss of generality. Thus there exists $U \in X$ such that $(A+$ $E) U=P^{*}$. Therefore,

$$
\left\langle P^{*}, Q\right\rangle=\langle(A+E) U, Q\rangle=\left\langle U,(A+E)^{*} Q\right\rangle=0 .
$$

However,

$$
\left\langle P^{*}, Q\right\rangle=\sum_{i=1}^{N} P_{0 i}+\sum_{j=1}^{2} \sum_{i=1}^{N} \int_{0}^{\infty} P_{j i}(x) d x>0 .
$$

Equation (30) contradicts (31). Then the algebraic index of 0 in $X$ is one. Then the algebraic multiplicity of 0 in $X$ is one. The proof of Theorem 6 is complete.

Lemma 5 and Theorem 6 can imply that several important results hold. Firstly, they imply that the spectral bound $s(A+E)$ of $A+E$ is zero. Secondly, Lemma 5 and Theorem 6 illustrate 0 is a strictly dominant eigenvalue of the operator $A+E$.

The following task is to verify the operator $A+E$ generates some $c_{0}$ semigroups $T(t)$.
Theorem 7. The system operator $A+E$ generates a positive contraction $c_{0}$ semigroup $T(t)$.

Proof. We can get the proof of Theorem 7 by the Phillips theorem (see [25]), Lemma 4, and Lemma 5.

Theorem 8. The system (15) has a unique nonnegative timedependent solution $P(\cdot, t)$, which satisfies $\|P(\cdot, t)\|=1$, for all $t \in[0, \infty)$.

Proof. In view of Theorem 7 and [25], we have that the system (15) has a unique nonnegative solution $P(\cdot, t)$ and it can be expressed as

$$
P(\cdot, t)=T(t) P_{0}, \quad \forall t \in[0, \infty) .
$$

Because $P(\cdot, t)$ satisfies $(2)-(4)$, it is easy to receive that $d\|P(\cdot, t)\| / d t=0$. Here $\|P(\cdot, t)\|=\left\|T(t) P_{0}\right\|=\left\|P_{0}\right\|=1$, for all $t \in[0, \infty)$.

Because $P_{0} \notin D(A),(32)$ is the mild solution of the system. However, Theorem 1 implies that the classical solution of the system uniquely exists for $t>0$. Hence, the mild solution $P(\cdot, t)=T(t) P_{0}$ is just the classical one for $t>0$. Thus the abstract Cauchy problem (15) is well posed.

Theorem 9. The time-dependent solution of the system (2)(4) strongly converges to its steady-state solution. That is, $\lim _{t \rightarrow \infty} P(\cdot, t)=P^{*}$, where $P^{*}$ is the eigenvector corresponding to 0 in $X$ satisfying $\left\|P^{*}\right\|=1$.

Proof. In the light of Theorem 8, the nonnegative solution of the system (2)-(4) can be expressed as $P(\cdot, t)=T(t) P_{0}$, for all $t \in[0, \infty$ ). Thus combing Theorem 2.10 (see [19]) together with Theorem 12.3 in [26], we can deduce that

$$
P(\cdot, t)=T(t) P_{0}=\left\langle P_{0}, Q\right\rangle P^{*}+R(t) P_{0}=P^{*}+R(t) P_{0},
$$

where $Q$ is the same as defined in Theorem 6 and $R(t)=C e^{-\varepsilon t}$ for suitable constants $\varepsilon>0$ and $C>0$. Hence we have

$$
\lim _{t \rightarrow \infty} P(\cdot, t)=\left\langle P_{0}, Q\right\rangle P^{*}=P^{*} .
$$

As a result, the exponential stability of the solution of the studied system was obtained.

Thus we show that the studied system has exponential stability. Exponential stability is a very important property in reliability study. We can overcome some problems readily and deduce some better conclusions by using it. For example, by using the property, the governors can make up their mind how to arrange the repairman to do minimal repair or overhaul in his work time to increase the profit of the system benefit.

As far as such a problem is concerned, previous literatures such as [27] only pointed out to when the profit of the system benefit with repairman vacation in steady state is larger than that of the classical system benefit. But this is a less practical condition because they cannot solve the following problems. Firstly, how long time the system will take to get the stability state. Secondly, whether the steady-state indices 
such as steady-state availability can substitute for the transient ones. Thirdly, what is the probability that the repairman can carry out minimal repair.

However, by studying the exponential stability of the system, all these problems can be solved easily. Actually, for a given fault, the system can get to the steady state at a very fast speed and its steady-state availability can substitute for the dynamic one by considering a safety factor. Moreover, $P_{0 i}(t)(i=1,2, \ldots, N)$ means the probability that the system is operating normally after every minimal repair, and the repairman is on vacation at time $t \geq 0$ and $P_{0 i}(t) \rightarrow P_{0 i}>0$; here $P_{0 i}(i=1,2, \ldots, N)$ is the first $N$ coordinate of the eigenvector $P^{*}$ in Theorem 9 . Then Theorem 9 indicates that, after a certain time $t>0$, the repairman can always be urged to overhaul with a fixed probability to increase the total profit of the system benefit.

\section{Reliability Indices}

In this section, we first present the steady-state probability and frequency of failure of the system with traditional method. Second, we propose the steady-state availability and the failure frequency of the system with one new method different from the traditional one (see [28]). And the two methods were compared; we have the second method is more practical and simple.

Firstly, the above equations (2) are valid for any $t \geq 0$. Since we are interested in the steady-state behavior of our system, we will seek the long-run probabilities which are the solution of the following equations obtained from (2) taking the limits as $t \rightarrow \infty$ :

$$
\begin{aligned}
& \left(\lambda_{h}+\lambda_{s}\right) P_{01}=\int_{0}^{\infty} P_{11}(x) \mu_{1}(x) d x+\int_{0}^{\infty} P_{2 N}(x) \mu_{3}(x) d x, \\
& \left(\lambda_{h}+a^{i-1} \lambda_{s}\right) P_{0 i}=\int_{0}^{\infty} P_{1 i}(x) \mu_{1}(x) d x \\
& +\int_{0}^{\infty} P_{2(i-1)}(x) \mu_{2}(x) d x \\
& i=2,3, \ldots, N \text {, } \\
& \left(\frac{d}{d x}+\mu_{1}(x)\right) P_{1 i}(x)=0, \quad i=1,2, \ldots, N \\
& \left(\frac{d}{d x}++\mu_{2}(x)\right) P_{2 i}(x)=0, \quad i=1,2, \ldots, N-1, \\
& \left(\frac{d}{d x}+\mu_{3}(x)\right) P_{2 N}(x)=0 .
\end{aligned}
$$

Equations (35) are to be solved under the following conditions:

$$
\begin{gathered}
P_{1 i}(0)=\lambda_{h} P_{0 i}, \quad i=1,2, \ldots, N, \\
P_{2 i}(0)=a^{i-1} \lambda_{s} P_{0 i}, \quad i=1,2, \ldots, N .
\end{gathered}
$$

The steady-state probabilities are $P_{0 i}, P_{1 i}=\int_{0}^{+\infty} P_{1 i}(x) d x$, $P_{2 i}=\int_{0}^{+\infty} P_{2 i}(x) d x$, where $i=1,2, \ldots, N$, respectively. The steady-state probabilities must satisfy the total probability equation

$$
\sum_{i=1}^{N}\left(P_{0 i}+P_{1 i}+P_{2 i}\right)=1
$$

Probability and frequency of failure are given by $P_{f}=$ $\sum_{i=1}^{N}\left(P_{1 i}+P_{2 i}\right)$ and $F_{f}=\sum_{i=1}^{N}\left(\lambda_{h}+a^{i-1} \lambda_{s}\right) P_{0 i}$.

Next, we obtain the steady-state availability and the failure frequency of the system by using the proposed method in this paper.

Theorem 10. The steady-state availability after the Nth time repair is

$$
A_{v N}=\frac{\sum_{i=1}^{N} a^{i-1}}{\sum_{i=1}^{N} a^{i-1}\left(1+\lambda_{h} E_{1}\right)+a^{N-1} \lambda_{s}\left[(N-1) E_{2}+E_{3}\right]},
$$

where $E_{i}=\int_{0}^{\infty} e^{-\int_{0}^{x} \mu_{i}(s) d s} d x(i=1,2,3)$.

Proof. Let

$$
\begin{aligned}
M & =\sum_{j=0}^{2} \sum_{i=1}^{N} P_{j i}^{*} \triangleq \sum_{i=1}^{N} P_{0 i}^{*}+\sum_{i=1}^{N} \int_{0}^{\infty} P_{1 i}^{*}(x) d x+\sum_{i=1}^{N} \int_{0}^{\infty} P_{2 i}^{*}(x) d x \\
& =\left\{\sum_{i=1}^{N} a^{i-1}\left(1+\lambda_{h} E_{1}\right)+a^{N-1} \lambda_{s}\left[(N-1) E_{2}+E_{3}\right]\right\} P_{0 N}^{*},
\end{aligned}
$$

where $P_{0 i}^{*}, P_{j i}^{*}(x)(j=1,2, i=1,2, \ldots, N)$ are the corresponding coordinates of $P^{*}$ presented in (29). Then the steady-state availability of the system can be expressed as

$$
\begin{aligned}
A_{v N} & =\frac{\sum_{i=1}^{N} P_{0 i}^{*}}{\sum_{i=1}^{N}\left(P_{0 i}^{*}+P_{1 i}^{*}(x)+P_{2 i}^{*}(x)\right)} \\
& =\frac{\sum_{i=1}^{N} a^{i-1}}{\sum_{i=1}^{N} a^{i-1}\left(1+\lambda_{h} E_{1}\right)+a^{N-1} \lambda_{s}\left[(N-1) E_{2}+E_{3}\right]}
\end{aligned}
$$

where $E_{i}=\int_{0}^{\infty} e^{-\int_{0}^{x} \mu_{i}(s) d s} d x(i=1,2,3)$.

From the above availability expression, the system steadystate availability decreases with the number of the minimal repair times increasing. So, the system steady-state availability is gradually decreasing (for $a>1$ ).

Theorem 11. The steady-state failure frequency after the $\mathrm{Nth}$ time repair is

$$
W_{f N}=\frac{\lambda_{h} \sum_{i=1}^{N} a^{i-1}+\lambda_{s} N a^{N-1}}{\sum_{i=1}^{N} a^{i-1}\left(1+\lambda_{h} E_{1}\right)+a^{N-1} \lambda_{s}\left[(N-1) E_{2}+E_{3}\right]},
$$

where $E_{i}=\int_{0}^{\infty} e^{-\int_{0}^{x} \mu_{i}(s) d s} d x(i=1,2,3)$. 

[28].

The proof of Theorem 11 is the same as Theorem 4.1 in

Of course, we can receive the formulations of the instantaneous reliability indices and their corresponding steady-state values as well, such as the reliability of the system and the probability of the repairman being busy.

As we all know, the reliability indices are ordinarily obtained by the Tauberian theorem and Laplacian transformation. However, the proposed method in this paper is probably more simply and more valuable in some practice applications, because the only thing needs to be considered is the eigenvector corresponding to eigenvalue 0 of the system operator.

In the light of these two methods, the first method is more idealistic and does not exist in real life. Compared with the first method, the second method is more practical and simple.

\section{Conclusion}

In this paper, we dealt with a repairable computer system which composed of hardware and software in series. We were dedicated to studying the unique existence and the exponential stability of the solution of the system. The exponential stability of the system guaranteed that the stability of the system was not easy to be affected by some factors such as failure rate and repair rate. And we presented a new method to receive the steady-state indices of the system by using the eigenvector corresponding to eigenvalue 0 of the system operator. It was more simple and practical than the traditional one.

However, it was well known that it was difficult or even impossible to obtain the time-dependent solution and the dynamic indices of the system. This paper presented a new method to overcome these problems from the view point of theory.

\section{Acknowledgments}

The authors thank the referees for their useful comments and suggestions. The research is supported by NSFC (no. 11201037) and Natural Science Foundation of Heilongjiang Province, China (no. QC2010024).

\section{References}

[1] T. S. S. Rao and U. C. Gupta, "Performance modelling of the M/G/1 machine repairman problem with cold-,warm- and hotstandbys," Computers \& Industrial Engineering, vol. 38, pp. 251267, 2009.

[2] E. J. Vanderperre and S. S. Makhanov, "On Gaver's parallel system sustained by a cold standby unit and attended by two repairmen," Operations Research Letters, vol. 30, no. 1, pp. 4348, 2002.

[3] K. H. Wang and J. C. Ke, "Probabilistic analysis of a repairable system with warm standbys plus balking and reneging," Applied Mathematical Modelling, vol. 27, pp. 327-336, 2003.

[4] Y. L. Zhang and G. J. Wang, "A deteriorating cold standby repairable system with priority in use," European Journal of Operational Research, vol. 183, pp. 278-295, 2007.
[5] M. J. Armstrong, "Age repair policies for the machine repair problem," European Journal of Operational Research, vol. 138, no. 1, pp. 127-141, 2002.

[6] H. B. Xu and J. M. Wang, "Asymptotic stability of software system with rejuvenation policy," in Proceedings of the 26th Chinese Control Conference, pp. 646-650, 2007.

[7] R. E. Barlow and F. Proschan, Statistical Theory of Reliability and lLife Testing, Holt, Reinehart and Winston, New York, NY, USA, 1975.

[8] Z. S. Khalil, "Availability of series system with various shut-off rules," IEEE Transactions on Reliability, vol. 34, pp. 187-189, 1985.

[9] A. B. Mark and M. M. Christine, "Developing integrated hardware-software reliability models: difficulties and issues," in AIAA/IEEE Digital Avionics Systems Conference-Proceedings, 1995.

[10] Y. L. Zhang and G. J. Wang, "A geometric process repair model for a series repairable system with $\mathrm{k}$ dissimilar components," Applied Mathematical Modelling, vol. 31, pp. 1997-2007, 2007.

[11] F. Rao, P. Q. Li, Y. P. Yao et al., "Hardware/software reliability growth model," Acta Automation Sinica, vol. 22, no. 1, pp. 3339, 1996.

[12] Y. Lin, "Geometric processes and replacement problem," Acta Mathematicae Applicatae Sinica, vol. 4, no. 4, pp. 366-377, 1988.

[13] Z. Shi, He He, and Z. Wu, "Software reliability and its evaluation," Computer Applications, vol. 20, no. 11, pp. 1-5, 2000.

[14] X. Wu, J. Zhang, Y. H. Tang, and B. Dong, "Based on the software and hardware features the reliability analysis of the computer system," Journal of Civil Aviation Flight University of China, vol. 17, no. 1, pp. 33-36, 2006.

[15] W.-W. Hu, H.-B. Xu, and G.-T Zhu, "Exponential stability of a parallel repairable system with warm standby," Acta Analysis Functionalis Applicata, vol. 9, no. 4, pp. 311-319, 2007.

[16] W. W. Hu, H. B. Xu, J. Y. Yu, and G. T. Zhu, "Exponential stability of a reparable multi-state device," Journal of Systems Science \& Complexity, vol. 20, no. 3, pp. 437-443, 2007.

[17] M. E. Gurtin and R. C. MacCamy, "Non-linear age-dependent population dynamics," Archive for Rational Mechanics and Analysis, vol. 54, pp. 281-300, 1974.

[18] J. H. Cao and K. Cheng, Introduction to Reliability Mathematics, Higher Education Press, Beijing, China, 2006.

[19] R. Nagel, One-Parameter Semigroups of Positive Operators, Lecture Notes in Mathematics, Springer, New York, NY, USA, 1986.

[20] G. Geni, X. Z. Li, and G. T. Zhu, Functional Analysis Method in Queueing Theory, Research Information Ltd, Hertfordshire, UK, 2001.

[21] F. Zheng and X. Qiao, "Exponential stability of a repairable system with $\mathrm{N}$ failure modes and one standby unit," in Proceedings of the International Conference on Intelligent Human-Machine Systems and Cybernetics, vol. 2, no. 5336023, pp. 146-149, 2009.

[22] W. Yuan and G. Xu, "Spectral analysis of a two unit deteriorating standby system with repair," WSEAS Transactions on Mathematics, vol. 10, no. 4, pp. 125-138, 2011.

[23] N. Dundord and J. T. Schwartz, Linear Operators, Part I, Wiley, New York, NY, USA, 1958.

[24] X. Qiao, D. Ma, and Z. X. Li, "Exponential asymptotic stability of repairable system with randomly selected repairman," in Proceedings of the Chinese Control and Decision Conference, no. 5191527, pp. 4580-4584, 2009.

[25] A. Pazy, Semigroups of Linear Operators and Applications to Partial Differential Equations, Springer, New York, NY, USA, 1983. 
[26] A. E. Taylor and D. C. Lay, Introduction to Functional Analysis, John Wiley \& Sons, New York, NY, USA, 1980.

[27] R. B. Liu, Y. H. Tang, and X. Y. Luo, "An n-unit series repairable system with a repairman doing other work," Journal of Natural Science of Heilongjiang University, vol. 22, no. 4, pp. 493-496, 2005.

[28] X. Qiao, D. Ma, Z. X. Zhao, and F. Z. Sun, "The indices analysis of a repairable computer system reliability," Advances in Electrical Engineering and Automation, AISC, vol. 139, pp. 299-305, 2011. 


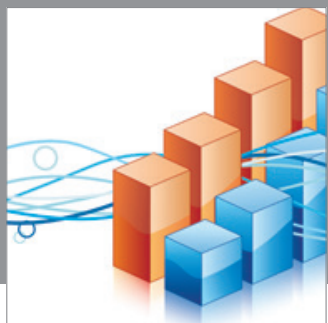

Advances in

Operations Research

mansans

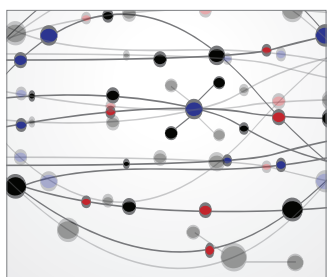

The Scientific World Journal
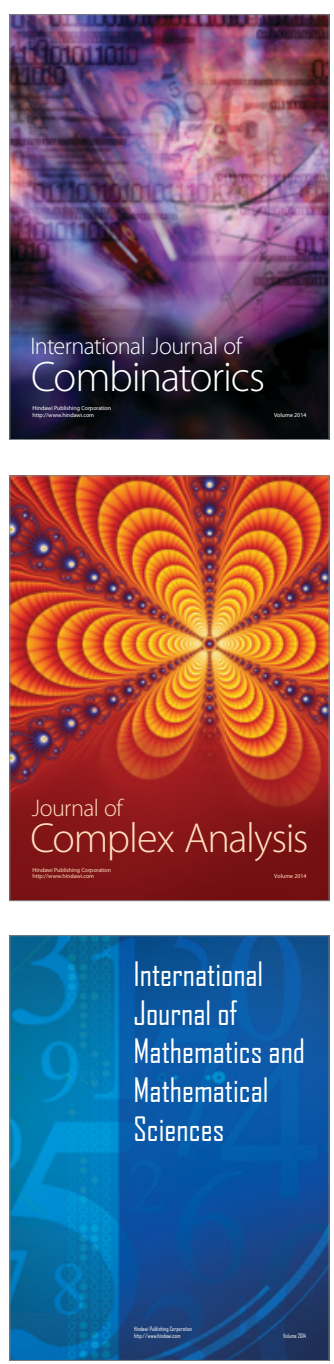
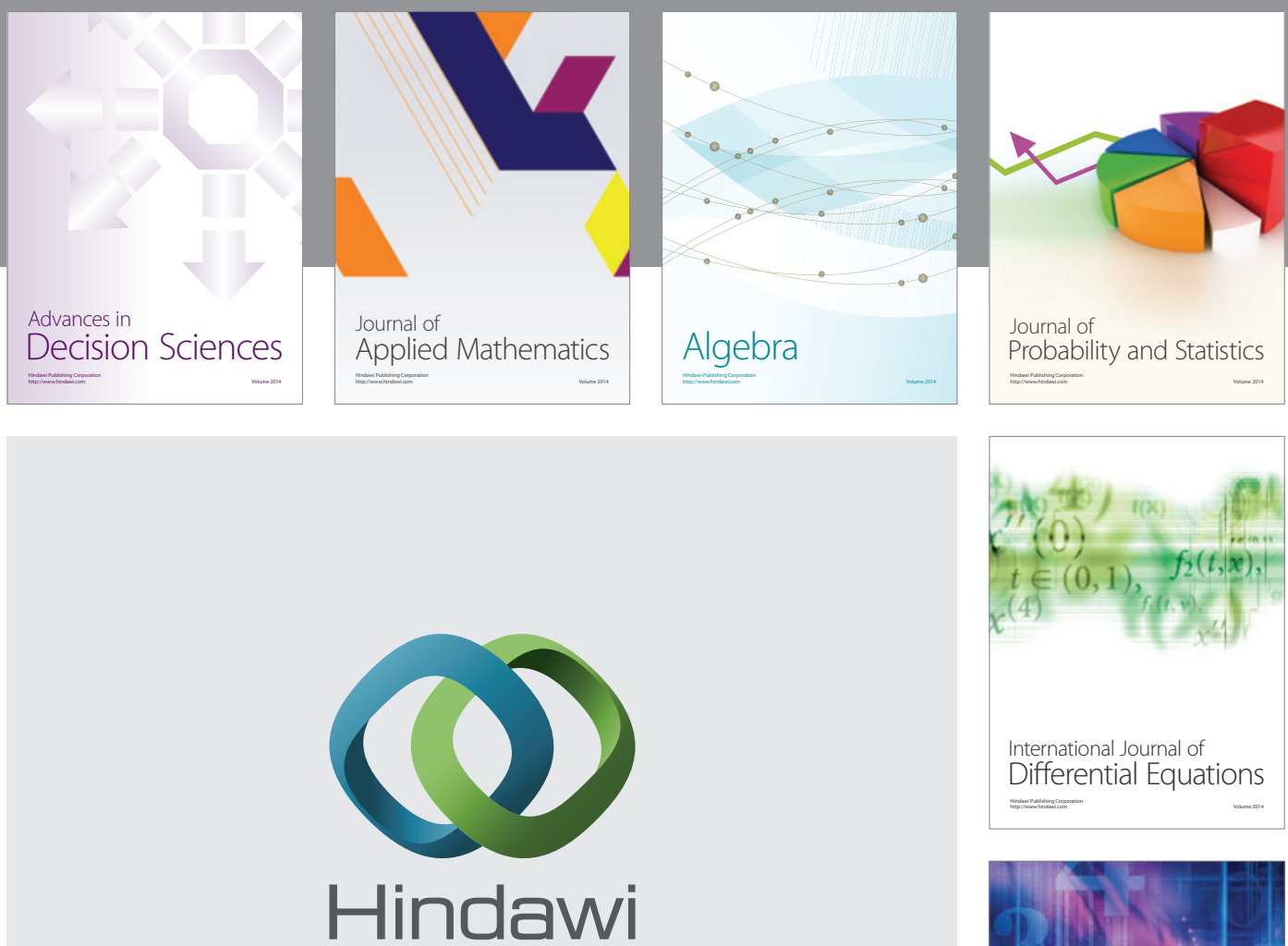

Submit your manuscripts at http://www.hindawi.com
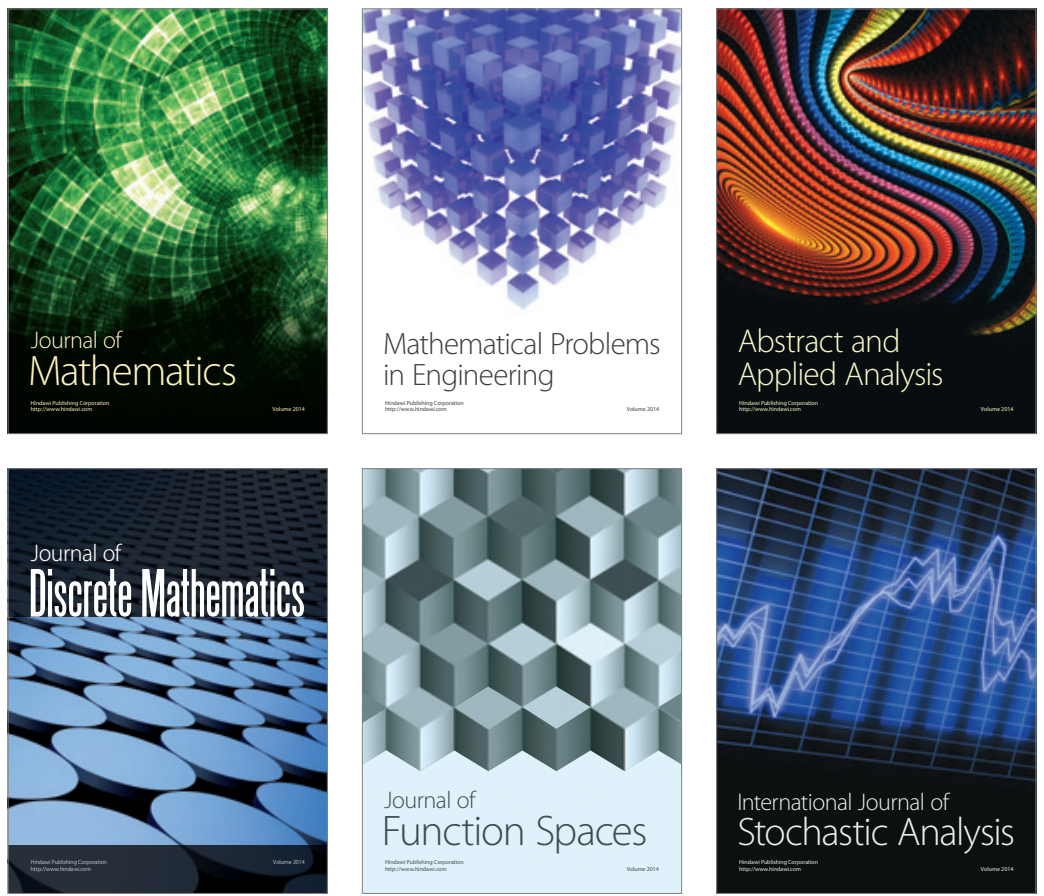

Journal of

Function Spaces

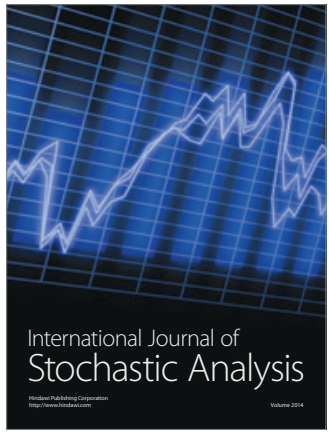

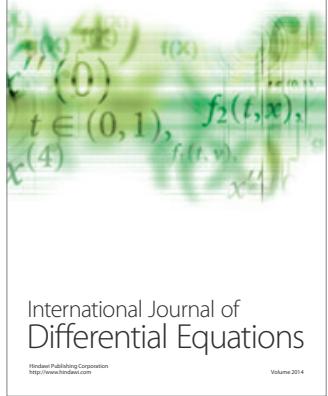
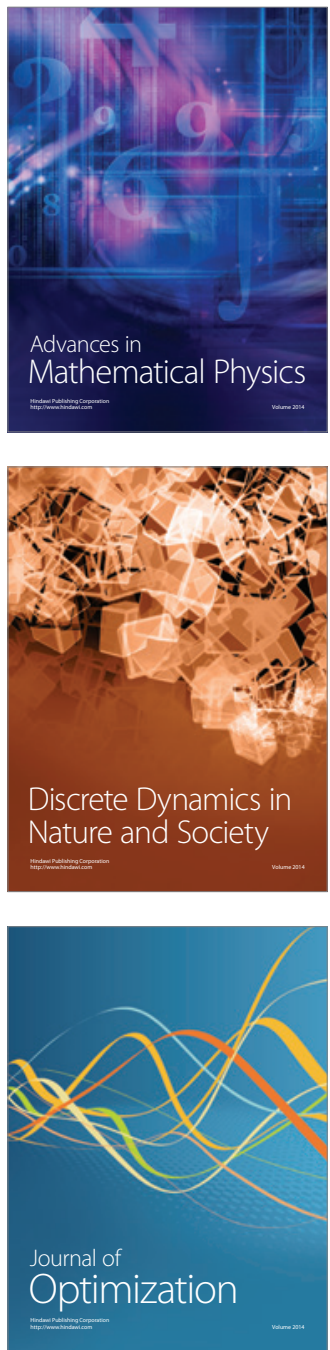